RAIRO Operations Research

RAIRO Oper. Res. 38 (2004) 153-171

DOI: $10.1051 /$ ro:2004017

\title{
OPTIMAL CONTROL FOR A BMAP/SM/1 QUEUE WITH MAP-INPUT OF DISASTERS AND TWO OPERATION MODES
}

\author{
Olga V. Semenova ${ }^{1}$
}

\begin{abstract}
A single-server queueing system with a batch Markovian arrival process (BMAP) and MAP-input of disasters causing all customers to leave the system instantaneously is considered. The system has two operation modes, which depend on the current queue length. The embedded and arbitrary time stationary queue length distribution has been derived and the optimal control threshold strategy has been determined.
\end{abstract}

Keywords. Negative arrivals, BMAP/SM/1 queue, Markovian arrival process of disasters, operation modes.

\section{INTRODUCTION}

Models of queueing systems describe quite adequately the situations appearing during queue serving in industry, communications, etc. Although having been investigated well by now, classical models of queueing systems fail to take into account the possibilities for time-changing system rates and disaster appearance considerably limiting their application.

During real queueing system operation the appearance of disasters is possible. Such queueing systems models describe disasters, which instantaneously destroy the entire queue including the customer being processed. Such disasters are the special case of the so called negative arrivals. The theory of negative arrivals has been originated and developed significantly by Gelenbe et al. [10-22]. The negative

\footnotetext{
1 Laboratory of Applied Probabilistic Analysis, Faculty of Applied Mathematics and Computer Sciences, Belarus State University, 4 F. Skorina Ave., 220050 Minsk 50, Belarus; e-mail: semenovaov@bsu.by
} 
arrival removes one customer $[14,15,18,24]$ or a batch of ones of random size [19] from the queueing system. The detailed survey of results concerning the queues and networks with negative customers is given by Artalejo [1].

A disaster as a partial case of a negative arrival removes all the customers from the system. So, the models with disasters can be considered as the partial case of models where a random number of customers is deleted by the negative arrival (see paper [19] by Gelenbe), if we assume that the infinite number of customers is removed from the system by the negative arrival with probability 1. Queueing systems with disasters were investigated in $[2,5-7,25]$.

The appearance of new practical problems in the last decades encouraged investigation of queueing systems with controlled rates of operation. The queueing system with controllable service rate belongs to the same class. Such queueing models have many promising applications for telecommunication systems and digital networks. It explains the attention that these models receive in literature. See for example [3,4,29-31]. Since there are many different types of data with different requirements to quality of service and different economical values, it is necessary to organize a dynamic schedule of information transmission. Controllable queueing models provide appropriate tools for optimizing such dynamic schedules. When the quality of the customers being processed is evaluated by some economic criterion, which includes holding and service costs and one customer loss cost, the problem of the modes switching in dependence on the current value of the queue length is very important and interesting.

Considering the batch Markovian arrival process (BMAP) as a customer input process is explained by its adequate describing of real information flows in modern communication network.

The rest of the paper is organized as follows. In Section 2, the model is described. The stationary queue length distribution at the customer departure epoch is derived in Section 3. The probability of successful service of a customer and the average interdeparture time are given in Section 4. In Section 5, the queue length distribution at an arbitrary time is obtained. The value of the cost criterion is calculated in Section 6. In Section 7, numerical examples are presented. Section 8 contains concluding remarks.

\section{MODEL}

Let us consider a single-server queue with unlimited waiting space having two possible modes of operation and additional input of disasters.

The $r$ th mode of customer processing is described as follows. The input into the system is a BMAP (Batch Markovian Arrival Process). This input is controlled by continuous-time Markov chain $\nu_{t}$ which is called a directing process of the BMAP. The state space of $\nu_{t}$ is $\{0,1, \ldots, W\}$. The transitions of process $\nu_{t}$ and arrivals of customers are performed according to a matrix generating function $D^{(r)}(z)=\sum_{k=0}^{\infty} D_{k}^{(r)} z^{k},|z|<1$. We suppose that the matrix $D^{(r)}(z)$ satisfies all assumptions of Lucantoni [26]. 
Denote by $\vec{\varphi}^{(r)}$ the stationary probability row vector of the Markov chain $\nu_{t}$. It is defined by equations:

$$
\vec{\varphi}^{(r)} D^{(r)}(1)=\vec{O}_{W+1}, \quad \vec{\varphi}^{(r)} \mathbf{1}_{W+1}=1 .
$$

Here $\vec{O}_{W+1}$ is a null vector of size $W+1$ and $\mathbf{1}_{W+1}=(1,1, \ldots, 1)^{T}$.

The intensity $\lambda^{(r)}$ of BMAP-input (the fundamental rate) is calculated as

$$
\lambda^{(r)}=\left.\vec{\varphi}^{(r)} \frac{\mathrm{d} D^{(r)}(z)}{\mathrm{d} z}\right|_{z=1} \mathbf{1}_{W+1} .
$$

We assume that successful service times of customers are the sojourn times of a semi-Markovian process $m_{t}$. This process has a state space $\{1, \ldots, M\}$ and a semiMarkovian kernel $B^{(r)}(x)$, where $B^{(r)}(x)$ is a matrix with entries $B_{m, m^{\prime}}^{(r)}(x), m, m^{\prime}=$ $\overline{1, M}$. The function $B_{m, m^{\prime}}^{(r)}(x)$ is the conditional distribution function of the sojourn time of the process $m_{t}$ in a state $m$ under the condition that the next state is $m^{\prime}$. We use the same assumptions about the kernel $B^{(r)}(x)$ as Neuts [28] and Lucantoni and Neuts [27]. Denote by $P^{(r)}=B^{(r)}(\infty)$ the transition matrix of the embedded Markov chain for the semi-Markovian process $m_{t}$. It is assumed that the states of the service directing process $m_{t}$ change according to the matrix $P^{(r)}$ at service completion epochs regardless of whether service is completed successfully or is canceled by a disaster appearance.

The arrival of disasters to the system is directed by a continues-time Markov chain $\eta_{t}$ with a state space $\{0,1, \ldots, N\}$ and a matrix transition generating function $F^{(r)}(z)=F_{0}^{(r)}+F_{1}^{(r)} z,|z| \leq 1$.

Transitions of the chain $\eta_{t}$ without generating of disasters are governed by the matrix $F_{0}^{(r)}$. Transitions of the chain $\eta_{t}$, which cause the appearance of disasters, are governed by the matrix $F_{1}^{(r)}, r=\overline{1,2}$.

Following Jain and Sigman [25] we suppose that the arrival of disasters immediately removes all customers from the system. If the system is empty at a disaster arrival epoch, this disaster is ignored by the system.

The changing of the operation mode is possible at customer departure epoch.

We assume that the quality of the system operation is evaluated by the following cost criterion:

$$
C=a \Lambda L+c_{1} \Phi_{1}+c_{2} \Phi_{2}+g R
$$

where $L$ is an average queue length at customer departure epoch; $\Lambda^{-1}$ is the average interdeparture time; $\Phi_{r}$ is the average fraction of time, when the $r$ th 
mode is used, $r=\overline{1,2}$; $\mathrm{R}$ is the average number of customers lost per time unit; $a, c_{1}, c_{2}$ and $g$ are the corresponding cost coefficients. We suppose that $a>0, c_{1} \leq$ $c_{2}, g \geq 0$.

For M/G/1 systems without disasters it has been proven (Tijms, [33]) that optimal strategy for the modes control is threshold strategy. Although the optimality of such strategies in class of all Markovian strategies for the system with disasters is not proved yet, it seems reasonable to exploit threshold strategy for the control of operation modes. The strategy is determined as follows. An integer-valued threshold $j$ is fixed, $j \geq 0$. If a queue length is $i$ at a given customer departure epoch and it does not exceed $j$, the first mode is selected for the next customer servicing. Otherwise, the second mode will be used. Also we assume that the first customer arriving to the system at the beginning of a system busy period is served always in the first mode.

Our problem is the optimal threshold strategy determining. We use a so-called direct approach, which is described as follows. Fix the threshold $j$. Calculate the stationary state probabilities of the Markov chain describing the system behavior. Using these probabilities, calculate the value of cost criterion under the fixed value of threshold and solve the problem of minimizing the cost criterion function of one integer-valued variable.

\section{Stationary QUeUe LENGTH Distribution}

Let the threshold $j$ be fixed, $j \geq 0$ and let $t_{n}$ be the $n$th epoch of customer departure from the system. Note that it is a service completion epoch or a disaster arrival epoch at a busy period. Consider the following four-dimensional process:

$$
\left\{i_{n}, \nu_{n}, \eta_{n}, m_{n}\right\}, n \geq 1
$$

where $i_{n}$ is a queue length at the epoch $t_{n}+0, i_{n} \geq 0 ; \nu_{n}$ is the state of arrival directing process $\nu_{t}$ at the epoch $t_{n}, \nu_{n}=\overline{0, W} ; \eta_{n}$ is the state of disasters directing process $\eta_{t}$ at the epoch $t_{n}, \eta_{n}=\overline{0, N}$ and $m_{n}$ is the state of service directing process $m_{t}$ at the epoch $t_{n}+0, m_{n}=\overline{1, M}$.

The process $\left\{i_{n}, \nu_{n}, \eta_{n}, m_{n}\right\}, n \geq 1$ is a four-dimensional embedded Markov chain. Enumerate the states of this chain in lexicographic order and denote by

$$
\begin{gathered}
P\left\{(i, \nu, \eta, m) \rightarrow\left(l, \nu^{\prime}, \eta^{\prime}, m^{\prime}\right)\right\} \\
=P\left\{i_{n+1}=l, \nu_{n+1}=\nu^{\prime}, \eta_{n+1}=\eta^{\prime},\right. \\
\left.m_{n+1}=m^{\prime} \mid i_{n}=i, \nu_{n}=\nu, \eta_{n}=\eta, m_{n}=m\right\}, \\
i, l \geq 0, \nu, \nu^{\prime}=\overline{0, W}, \eta, \eta^{\prime}=\overline{0, N}, m, m^{\prime}=\overline{1, M} .
\end{gathered}
$$


Lemma 1. The transitions probabilities $P\left\{(i, \nu, \eta, m) \rightarrow\left(l, \nu^{\prime}, \eta^{\prime}, m^{\prime}\right)\right\}$ form the following matrices:

$$
\begin{array}{ll}
\Omega_{l-i+1}^{(1)}, & \text { if } 0<i \leq j, l \geq i-1, \\
\Omega_{l-i+1}^{(2)}, & \text { if } i>j, l \geq i-1, \\
S^{(1)}, & \text { if } 1<i \leq j, l=0, \\
S^{(2)}, & \text { if } i>j, l=0, \\
\Omega_{0}^{(1)}+S^{(1)}, & \text { if } i=1, l=0, \\
\sum_{k=1}^{l+1} \Psi_{k} \Omega_{l-i+1}^{(1)}, & \text { if } i=0, l>0, \\
\Psi_{1} \Omega_{0}^{(1)}+\sum_{k=1}^{\infty} \Psi_{k} S^{(1)}, & \text { if } i=0, l=0, \\
O, & \text { if } l<i-1, l \neq 0 .
\end{array}
$$

Here:

- the entries of the matrix $\Omega_{l}^{(r)}, l \geq 0, r=\overline{1,2}$ have the following probabilistic sense: $l$ customers arrive but no disaster arrives to the system during the customer processing, which is performed in the $r$ th mode, and a triple $\left\{\nu_{n}, \eta_{n}, m_{n}\right\}$ transits from the state $\{\nu, \eta, m\}$ into the state $\left\{\nu^{\prime}, \eta^{\prime}, m^{\prime}\right\}$. These matrices can be determined from the following matrix expansion:

$\sum_{l=0}^{\infty} \Omega_{l}^{(r)} z^{l}=\int_{0}^{\infty} \mathrm{e}^{D^{(r)}(z) t} \otimes \mathrm{e}^{F_{0}^{(r)} t} \otimes \mathrm{d} B^{(r)}(t)=\hat{\beta}_{r}(z), \quad r=\overline{1,2}$.

The matrices $\Omega_{l}^{(r)}$ can be calculated in essentially the same way as the corresponding matrices in Lucantoni [26];

- the entries of the matrix $S^{(r)}, r=\overline{1,2}$ have the following probabilistic sense: a disaster appears during the current customer processing, which is performed in the $r$-th mode, and a triple $\left\{\nu_{n}, \eta_{n}, m_{n}\right\}$ transits from the state $\{\nu, \eta, m\}$ into the state $\left\{\nu^{\prime}, \eta^{\prime}, m^{\prime}\right\}$ during this uncompleted service. This matrix is determined by

$$
S^{(r)}=\int_{0}^{\infty} \mathrm{e}^{D^{(r)}(1) t} \otimes\left(\mathrm{e}^{F_{0}^{(r)}} F_{1}^{(r)}\right) \otimes\left(P^{(r)}-B^{(r)}(t)\right) \mathrm{d} t ;
$$

- the entries of the matrix $\Psi_{k}, k \geq 1$ have the following probabilistic sense: a busy period begins by the arrival of customers batch of size $k$ and a triple $\left\{\nu_{n}, \eta_{n}, m_{n}\right\}$ transits from the state $\{\nu, \eta, m\}$ into the state $\left\{\nu^{\prime}, \eta^{\prime}, m^{\prime}\right\}$ during the idle period. These matrices are defined by

$$
\begin{aligned}
\Psi_{k} & =\int_{0}^{\infty}\left(\mathrm{e}^{D_{0}^{(1)} t} D_{k}^{(1)}\right) \otimes \mathrm{e}^{F^{(1)}(1) t} \mathrm{~d} t \otimes I_{M} \\
& =-\left[\left(D_{0}^{(1)} \oplus F^{(1)}(1)\right)^{-1}\left(D_{k}^{(1)} \otimes I_{N+1}\right)\right] \otimes I_{M}, k \geq 1 .
\end{aligned}
$$

Here $\otimes$ and $\oplus$ are the symbols of the Kronecker product and the Kronecker sum, respectively; see for example [23] for definitions and properties. $I_{i}$ denotes an 
identity matrix of the size $i, I$ later denotes an identity matrix of the size $K$, where $K=(W+1)(N+1) M$. $O$ is the zero matrix.

Introduce into consideration the stationary state probabilities

$$
\begin{aligned}
\pi(i, \nu, \eta, m)=\lim _{n \rightarrow \infty} P\left\{i_{n}=i, \nu_{n}=\nu, \eta_{n}\right. & \left.=\eta, m_{n}=m\right\}, \\
i & \geq 0, \nu=\overline{0, W}, \eta=\overline{0, N}, m=\overline{1, M} .
\end{aligned}
$$

Note that due to the presence of disasters, the limits (5) exist for any finite positive arrival and service rates.

Denote

$$
\begin{aligned}
\vec{\pi}(i, \nu, \eta) & =(\pi(i, \nu, \eta, 1), \ldots, \pi(i, \nu, \eta, M)), \\
\vec{\pi}(i, \nu) & =(\vec{\pi}(i, \nu, 0), \ldots, \vec{\pi}(i, \nu, N)) \\
\vec{\pi}_{i} & =(\vec{\pi}(i, 0), \ldots, \vec{\pi}(i, W)) .
\end{aligned}
$$

Introduce also the partial generating functions

$$
\vec{\Pi}_{1}(z)=\sum_{i=0}^{j} \vec{\pi}_{i} z^{i}, \quad \vec{\Pi}_{2}(z)=\sum_{i=j+1}^{\infty} \vec{\pi}_{i} z^{i}, \quad|z|<1
$$

Theorem 1. The partial generating functions $\vec{\Pi}_{1}(z), \vec{\Pi}_{2}(z)$ satisfy the following matrix functional equation:

$\vec{\Pi}_{1}(z)\left(z I-\hat{\beta}_{1}(z)\right)+\vec{\Pi}_{2}(z)\left(z I-\hat{\beta}_{2}(z)\right)=\vec{\pi}_{0}(\Psi(z)-I) \hat{\beta}_{1}(z)+\vec{\pi}_{0}(\Psi-I) S^{(1)} z+\vec{\theta} z, \quad(6)$

where

$$
\begin{aligned}
\Psi(z) & =\sum_{k=1}^{\infty} \Psi_{k} z^{k}=-\left[\left(D_{0}^{(1)} \oplus F^{(1)}(1)\right)^{-1}\left(\left(D^{(1)}(z)-D_{0}^{(1)}\right) \otimes I_{N+1}\right)\right] \otimes I_{M}, \\
\Psi & =\Psi(1),
\end{aligned}
$$

$$
\vec{\theta}=\vec{\Pi}_{1}(1) S^{(1)}+\vec{\Pi}_{2}(1) S^{(2)},
$$

and matrices $\hat{\beta}_{r}(z), S^{(r)}, r=\overline{1,2}$ are determined by (2) and (3), respectively. 
Proof. Using the formula of total probability and Lemma 1, we derive the following system of equations:

$$
\begin{aligned}
\vec{\pi}_{0} & =\vec{\pi}_{0}\left(\Psi_{1} \Omega_{0}^{(1)}+\Psi S^{(1)}\right)+\vec{\pi}_{1} \Omega_{0}^{(1)}+\sum_{i=1}^{j} \vec{\pi}_{i} S^{(1)}+\sum_{i=j+1}^{\infty} \vec{\pi}_{i} S^{(2)}, \\
\vec{\pi}_{i} & =\vec{\pi}_{0} \sum_{k=1}^{i+1} \Psi_{k} \Omega_{i-k+1}^{(1)}+\sum_{k=1}^{i+1} \vec{\pi}_{k} \Omega_{i-k+1}^{(1)}, \quad i=\overline{1, j-1}, \\
\vec{\pi}_{j+l} & =\vec{\pi}_{0} \sum_{k=1}^{j+l+1} \Psi_{k} \Omega_{j+l-k+1}^{(1)}+\sum_{k=1}^{j} \vec{\pi}_{k} \Omega_{j+l-k+1}^{(1)}+\sum_{k=1}^{l+1} \vec{\pi}_{j+k} \Omega_{l-k+1}^{(2)}, \quad l \geq 0 .
\end{aligned}
$$

Multiplying the equations (9) by a corresponding degree of $z$, summing up and taking into account notation (8) we derive the equation (6). Formula (7) follows from (4) and Theorem 1 is proved.

Corollary 1. The generating function $\vec{\Pi}_{1}(z)$ is determined by the equation:

$$
\vec{\Pi}_{1}(z)=\vec{\pi}_{0} Y(z)+\left(\vec{\pi}_{0}(\Psi-I) S^{(1)}+\vec{\theta}\right) Q(z),
$$

where $Y(z)=\sum_{i=0}^{j} Y_{i} z^{i}, Q(z)=\sum_{i=0}^{j} Q_{i} z^{i}$, and matrices $Y_{i}$ and $Q_{i}, i=\overline{0, j}$ are calculated from the recurrent formulas:

$$
\begin{aligned}
& Y_{0}=I \\
& Y_{i+1}=\left(Y_{i}-\sum_{k=1}^{i+1} \Psi_{k} \Omega_{i-k+1}^{(1)}-\sum_{k=1}^{i} Y_{k} \Omega_{i-k+1}^{(1)}\right)\left(\Omega_{0}^{(1)}\right)^{-1}, \quad i=\overline{0, j-1} \\
& Q_{0}=O, \quad Q_{1}=-\left(\Omega_{0}^{(1)}\right)^{-1}, \\
& Q_{i+1}=\left(Q_{i}-\sum_{k=1}^{i} Q_{k} \Omega_{i-k+1}^{(1)}\right)\left(\Omega_{0}^{(1)}\right)^{-1}, \quad i=\overline{1, j-1} .
\end{aligned}
$$

Proof. Rewrite the relation (6) in the form

$$
\begin{aligned}
\vec{\Pi}_{1}(z)=\left[\vec{\pi}_{0}\left((\Psi(z)-I) \hat{\beta}_{1}(z)+(\Psi-I) S^{(1)} z\right)+\vec{\theta} z\right. \\
\left.+\vec{\Pi}_{2}(z)\left(\hat{\beta}_{2}(z)-z I\right)\right]\left(z I-\hat{\beta}_{1}(z)\right)^{-1}
\end{aligned}
$$

From (12) we derive the following equations for the vectors $\vec{\pi}_{i}, i=\overline{1, j}$ :

$$
\vec{\pi}_{i}=\vec{\pi}_{0} Y_{i}+\left[\vec{\pi}_{0}(\Psi-I) S^{(1)}+\vec{\theta}\right] Q_{i}, i=\overline{1, j}
$$


where

$$
\begin{aligned}
Y_{i} & =\left.\frac{1}{i !} \frac{\partial^{i}}{\partial z^{i}}(\Psi(z)-I) \hat{\beta}_{1}(z)\left(z I-\hat{\beta}_{1}(z)\right)^{-1}\right|_{z=0}, \\
Q_{i} & =\left.\frac{1}{i !} \frac{\partial^{i}}{\partial z^{i}}\left(z\left(z I-\hat{\beta}_{1}(z)\right)^{-1}\right)\right|_{z=0}, \quad i=\overline{0, j}
\end{aligned}
$$

Multiplying the equations (13) by a corresponding degree of $z$ and summing up, we get the formula (10).

By expanding the expressions $(\Psi(z)-I) \hat{\beta}_{1}(z)\left(z I-\hat{\beta}_{1}(z)\right)^{-1}$ and $z\left(z I-\hat{\beta}_{1}(z)\right)^{-1}$ in series at the point $z=0$ it can be shown that matrices $Y_{i}$ and $Q_{i}, i=\overline{0, j}$ satisfy the recursions (11). Corollary 1 is proved.

So the vector function $\vec{\Pi}_{1}(z)$ is a known function up to the vectors $\vec{\pi}_{0}$ and $\vec{\theta}$. Further we well get a relation between vectors $\vec{\pi}_{0}$ and $\vec{\theta}$.

By substituting $z=1$ into (6) and using the equation (8) we derive

$$
\vec{\Pi}_{2}(1)\left(I-A_{2}\right)=\vec{\pi}_{0}(\Psi-I) A_{1}+\vec{\Pi}_{1}(1)\left(A_{1}-I\right),
$$

where $A_{r}=\hat{\beta}_{r}(1)+S^{(r)}, r=\overline{1,2}$. It should be noted that $A_{1}$ and $A_{2}$ are the matrices of transition probabilities of the all directing processes during the service time. Hence these matrices are stochastic and the matrices $I-A_{r}, r=\overline{1,2}$ are singular. Therefore $A_{r}$ has a row eigenvector $\vec{\rho}_{r}$ corresponding to the eigenvalue 1:

$$
\vec{\rho}_{r} A_{r}=\vec{\rho}_{r}, \quad \vec{\rho}_{r} \mathbf{1}=1, \quad r=\overline{1,2}
$$

There is a well-known fact from the matrix theory that the matrix $I-A_{2}+\mathbf{1} \overrightarrow{\rho_{2}}$ is not singular. So matrix $Z=\left(I-A_{2}+\mathbf{1} \vec{\rho}_{2}\right)^{-1}$ exists. Since the vector $\vec{\Pi}_{1}(1)+\vec{\Pi}_{2}(1)$ is stochastic, adding the expression $\left(\vec{\Pi}_{1}(1)+\vec{\Pi}_{2}(1)\right) \mathbf{1} \vec{\rho}_{2}=\vec{\rho}_{2}$ to the both parts of equation (14) and multiplying the result by $Z$ from the right side we derive

$$
\vec{\Pi}_{2}(1)=\vec{\rho}_{2}+\vec{\pi}_{0}(\Psi-I) A_{1} Z+\vec{\Pi}_{1}(1)\left(A_{1}-I-\mathbf{1} \vec{\rho}_{2}\right) Z
$$

Substituting (15) and expression for the vector $\vec{\Pi}_{1}(1)$ from (10) in (8) we get the following result.

Corollary 2. The dependence of $\vec{\theta}$ on vector $\vec{\pi}_{0}$ has the form

$$
\vec{\theta}=\vec{\pi}_{0} T V+\vec{\rho}_{2} S^{(2)} V
$$

where

$$
\begin{aligned}
T & =\left[Y(1)+(\Psi-I) S^{(1)} Q(1)\right] A^{*}+(\Psi-I) A_{1} Z S^{(2)}, \\
V & =\left(I-Q(1) A^{*}\right)^{-1}, \\
A^{*} & =S^{(1)}+\left(A_{1}-I-\mathbf{1} \vec{\rho}_{2}\right) Z S^{(2)} .
\end{aligned}
$$


Rewrite (6) in the form

$$
\begin{aligned}
\Pi_{2}(z)\left(z I-\hat{\beta}_{2}(z)\right)=\vec{\pi}_{0}(\Psi(z)-I) \hat{\beta}_{1}(z)+\vec{\pi}_{0}(\Psi-I) S^{(1)} z+\vec{\theta} z \\
+\vec{\Pi}_{1}(z)\left(\hat{\beta}_{1}(z)-z I\right) .
\end{aligned}
$$

Substituting (10) and (16) in (17), we derive an algorithm for calculating the unknown vector $\vec{\pi}_{0}$.

It is known (Th. 3 in [9]) that the equation

$$
\operatorname{det}\left(z I-\hat{\beta}_{2}(z)\right)=0
$$

has exactly $K$ roots inside the unit disc $|z|<1$ and no root on the circle because the matrix $\hat{\beta}_{2}(z)$ is substochastic. Denote these roots as $z_{k}$ with corresponding multiplicities $n_{k}, k=\overline{1, H}, \sum_{k=1}^{H} n_{k}=K$, where $H$ is the number of different root.

The vector generating function $\vec{\Pi}_{2}(z)$ is analytic in the unit disc $|z|<1$. So entries of the unknown vector $\vec{\pi}_{0}$ satisfy the following system of linear algebraic equations:

$$
\begin{array}{r}
\vec{\pi}_{0} \frac{\mathrm{d}^{n}}{\mathrm{~d} z^{n}}\left\{\left[(\Psi(z)-I) \hat{\beta}_{1}(z)+Y(z)\left(\hat{\beta}_{1}(z)-z I\right)+\left((\Psi-I) S^{(1)}+T V\right)\right.\right. \\
\left.\left.\times\left(z I+Q(z)\left(\hat{\beta}_{1}(z)-z I\right)\right)\right] \operatorname{adj}\left(z I-\hat{\beta}_{2}(z)\right)\right\}\left.\right|_{z=z_{k}} \vec{e}_{1} \\
=-\left.\vec{\rho}_{2} S^{(2)} V \frac{\mathrm{d}^{n}}{\mathrm{~d} z^{n}}\left\{\left[z I+Q(z)\left(\hat{\beta}_{1}(z)-z I\right)\right] \operatorname{adj}\left(z I-\hat{\beta}_{2}(z)\right)\right\}\right|_{z=z_{k}} ^{\quad \vec{e}_{1},} \\
n=\overline{0, n_{k}-1}, \quad k=\overline{1, H} .
\end{array}
$$

Here $\vec{e}_{1}=(1,0, \cdots, 0)^{T},{ }^{T}$ denotes transposition and adj denotes the adjoint matrix. Following Gail et al. [8,9] it can be shown that (18) has unique solution.

Having known the value of vector $\vec{\pi}_{0}$, we calculate the value of $\vec{\theta}$. Then substituting the values of these vectors into (10) and (17) we get the final expressions for the partial generating functions $\vec{\Pi}_{1}(z)$ and $\vec{\Pi}_{2}(z)$.

\section{Probability of SucCessful Service of A Customer AND AVERAGE INTERDEPARTURE TIME}

Due to the presence of disasters, some of the customers leave the system unserved or only partially served. So let find the probability of successful service of an arbitrary customer. To this end we consider the five-dimensional Markov 
chain $\left\{i_{n}, \nu_{n}, \eta_{n}, m_{n}, c_{n}\right\}, n \geq 1$. Here the processes $\left\{i_{n}, \nu_{n}, \eta_{n}, m_{n}\right\}$ have the same sense as in the Section 3. The process $c_{n}, n \geq 1$ is defined as follows:

- $c_{n}=1$, if $t_{n}$ is the epoch of successful service completion;

- $c_{n}=0$, if $t_{n}$ is the disaster arrival epoch.

If $c_{n}=1$, we consider $i_{n}$ as the number of customers in the system at epoch $t_{n}+0, i_{n} \geq 0$. If $c_{n} \geq 1$, we consider $i_{n}$ as the number of customers that leave the system at epoch $t_{n}$ due to a disaster appearance, $i_{n} \geq 1$.

Introduce into consideration the stationary probabilities

$$
\begin{aligned}
p(i, \nu, \eta, m) & =\lim _{n \rightarrow \infty} P\left\{i_{n}=i, \nu_{n}=\nu, \eta_{n}=\eta, m_{n}=m, c_{n}=1\right\}, i_{n} \geq 0, \\
k(i, \nu, \eta, m) & =\lim _{n \rightarrow \infty} P\left\{i_{n}=i, \nu_{n}=\nu, \eta_{n}=\eta, m_{n}=m, c_{n}=0\right\}, i_{n} \geq 1, \\
\nu & =\overline{0, W}, \quad \eta=\overline{0, N}, \quad m=\overline{1, M} .
\end{aligned}
$$

Corresponding to the lexicographic order, introduce also the vectors

$$
\begin{aligned}
\vec{p}(i, \nu, \eta) & =(p(i, \nu, \eta, 1), \cdots, p(i, \nu, \eta, M)), \\
\vec{k}(i, \nu, \eta) & =(k(i, \nu, \eta, 1), \cdots, k(i, \nu, \eta, M)), \\
\vec{p}(i, \nu) & =(\vec{p}(i, \nu, 0), \cdots, \vec{p}(i, \nu, N)), \\
\vec{k}(i, \nu) & =(\vec{k}(i, \nu, 0), \cdots, \vec{k}(i, \nu, N)), \\
\vec{p}_{i} & =(\vec{p}(i, 0), \cdots, \vec{p}(i, W)), \\
\vec{k}_{i} & =(\vec{k}(i, 0), \cdots, \vec{k}(i, W))
\end{aligned}
$$

and generating functions

$$
\vec{P}_{1}(z)=\sum_{i=0}^{j} \vec{p}_{i} z^{i}, \quad \vec{P}_{2}(z)=\sum_{i=j+1}^{\infty} \vec{p}_{i} z^{i}, \quad \vec{K}(z)=\sum_{i=1}^{\infty} \vec{k}_{i} z^{i}, \quad|z| \leq 1 .
$$

Theorem 2. The vector generating functions $\vec{P}_{1}(z), \vec{P}_{2}(z)$ and $\vec{K}(z)$ satisfy the following system of matrix functional equations:

$$
\begin{aligned}
& \vec{P}_{1}(z)\left(z I-\hat{\beta}_{1}(z)\right)+\vec{P}_{2}(z)\left(z I-\hat{\beta}_{2}(z)\right)=-\vec{p}_{0} \hat{\beta}_{1}(z)+\left(\vec{p}_{0}+\vec{K}(1)\right) \Psi(z) \hat{\beta}_{1}(z) \\
& \vec{K}(z)=\left(\vec{p}_{0}(\Psi(z)-I)+\vec{K}(1) \Psi(z)+\vec{P}_{1}(z)\right) S^{(1)}(z)+\vec{P}_{2}(z) S^{(2)}(z)
\end{aligned}
$$

where

$$
S^{(r)}(z)=\sum_{l=0}^{\infty} S_{l}^{(r)} z^{l}=\int_{0}^{\infty} \mathrm{e}^{D^{(r)}(z) t} \otimes\left(\mathrm{e}^{F_{0}^{(r)} t} F_{1}^{(r)}\right) \otimes\left(P^{(r)}-B^{(r)}(t)\right) \mathrm{d} t,
$$

in particular $S^{(r)}(1)=S^{(r)}, r=\overline{1,2}$. 
Proof. The vectors of the stationary probabilities $\vec{p}_{i}, i \geq 0, \vec{k}_{i}, i \geq 1$ satisfy the following equations:

$$
\begin{aligned}
\vec{p}_{i}= & \left(\vec{p}_{0}+\sum_{m=1}^{\infty} \vec{k}_{m}\right) \sum_{n=1}^{i+1} \Psi_{n} \Omega_{i-n+1}^{(1)}+\sum_{n=1}^{i+1} \vec{p}_{n} \Omega_{i-n+1}^{(1)}, i=\overline{0, j-1}, \\
\vec{p}_{j+l}= & \left(\vec{p}_{0}+\sum_{m=1}^{\infty} \vec{k}_{m}\right) \sum_{n=1}^{j+l+1} \Psi_{n} \Omega_{j+l-n+1}^{(1)} \\
& +\sum_{n=1}^{j} \vec{p}_{n} \Omega_{j+l-n+1}^{(1)}+\sum_{n=1}^{l+1} \vec{p}_{j+n} \Omega_{l-n+1}^{(2)}, l \geq 0, \\
\vec{k}_{i}= & \left(\vec{p}_{0}+\sum_{m=1}^{\infty} \vec{k}_{m}\right) \sum_{n=1}^{i} \Psi_{n} S_{i-n}^{(1)}+\sum_{n=1}^{i} \vec{p}_{n} S_{i-n}^{(1)}, i=\overline{1, j}, \\
\vec{k}_{j+l}= & \left(\vec{p}_{0}+\sum_{m=1}^{\infty} \vec{k}_{m}\right) \sum_{n=1}^{j+l} \Psi_{n} S_{j+l-n}^{(1)}+\sum_{n=1}^{j} \vec{p}_{n} S_{j+l-n}^{(1)}+\sum_{k=1}^{l} \vec{p}_{j+k} S_{l-k}^{(2)}, l \geq 1,
\end{aligned}
$$

where $S_{m}^{(r)}, m \geq 0$ are the coefficients of the expansion (20) and they are the matrices of the transition probabilities of the process $\left\{\nu_{n}, \eta_{n}, m_{n}\right\}$ corresponding to the arrival of $m$ customers during the uncompleted service that is interrupted by a disaster arrival when the system operates in the $r$ th mode, $r=\overline{1,2}$. Multiplying the equations in the system (21) by the corresponding degree of $z$ and summing up, and using notion (20), we get the functional equations (19). So, Theorem 2 is proved.

It is easy to see that $\vec{\pi}_{0}=\vec{p}_{0}+\vec{K}(1)$. So the equations (19) can be rewritten as follows:

$$
\begin{gathered}
\vec{P}_{1}(z)\left(z I-\hat{\beta}_{1}(z)\right)+\vec{P}_{2}(z)\left(z I-\hat{\beta}_{2}(z)\right)=\left(\vec{\pi}_{0}(\Psi(z)-I)+\vec{K}(1)\right) \hat{\beta}_{1}(z), \\
\vec{K}(z)=\left(\vec{\pi}_{0}(\Psi(z)-I)+\vec{K}(1)+\vec{P}_{1}(z)\right) S^{(1)}(z)+\vec{P}_{2}(z) S^{(2)}(z) .
\end{gathered}
$$

By substituting $z=1$ into (23) and using the notion (8) we derive the following relation:

$$
\vec{K}(1)=\vec{\pi}_{0}(\Psi-I) S^{(1)}+\vec{\theta} .
$$

In particular the formula (10) can be rewritten as

$$
\vec{\Pi}_{1}(z)=\vec{\pi}_{0} Y(z)+\vec{K}(1) Q(z) .
$$

So, having known the values of vectors $\vec{\pi}_{0}$ and $\vec{\theta}$ we can find the value of the vector $\vec{K}(1)$ from (24). Hence the vector $\vec{p}_{0}$ can be calculated as $\vec{p}_{0}=\vec{\pi}_{0}-\vec{K}(1)$. From 
(22) and (23) we get the following equations:

$$
\begin{aligned}
\vec{P}_{1}(1)= & \vec{\pi}_{0} Y(1)+\vec{K}(1)[Q(1)-I], \\
\vec{P}_{2}(1)=\left\{\left[\vec{\pi}_{0}(\Psi-I)+\vec{K}(1)\right] \hat{\beta}_{1}(1)+\vec{P}_{1}(1)\left(\hat{\beta}_{1}(1)-I\right)\right\}\left(I-\hat{\beta}_{2}(1)\right)^{-1}, & \vec{P}_{1}^{\prime}(1)=\vec{\pi}_{0} Y^{\prime}(1)+\vec{K}(1) Q^{\prime}(1), \\
\vec{P}_{2}^{\prime}(1)=\left\{\vec{\pi}_{0}\left[\Psi^{\prime}(1) \hat{\beta}_{1}(1)+(\Psi-I) \hat{\beta}_{1}^{\prime}(1)\right]+\vec{K}(1) \hat{\beta}_{1}^{\prime}(1)+\vec{P}_{1}(1)\left(\hat{\beta}_{1}^{\prime}(1)-I\right)\right. & \left.\quad \vec{P}_{1}^{\prime}(1)\left(\hat{\beta}_{1}(1)-I\right)+\vec{P}_{2}(1)\left(\hat{\beta}_{2}^{\prime}(1)-I\right)\right\}\left(I-\hat{\beta}_{2}(1)\right)^{-1}, \\
\vec{K}^{\prime}(1)=\left[\vec{\pi}_{0} \Psi^{\prime}(1)+\vec{P}_{1}^{\prime}(1)\right] S^{(1)}+\left[\vec{\pi}_{0}(\Psi-I)+\vec{K}(1)+\vec{P}_{1}(1)\right]\left(S^{(1)}\right)^{\prime}(1) & \quad \vec{P}_{2}^{\prime}(1) S^{(2)}+\vec{P}_{2}(1)\left(S^{(2)}\right)^{\prime}(1) .
\end{aligned}
$$

Note that $\left(\vec{P}_{1}(1)+\vec{P}_{2}(1)\right) \mathbf{1}$ is the probability of having successful service completion of an arbitrary customer at a given customer departure epoch and $\vec{K}^{\prime}(1) \mathbf{1}$ is the average number of customers which are deleted from the system at a disaster arrival epoch.

Denote by $P_{+}$the probability of successful service of an arbitrary customer. Using the ergodic theorems for functionals defined on the Markov chain (see e.g. [32]), we derive the following result from the above considerations.

Theorem 3. The probability $P_{+}$of successful service of an arbitrary customer is defined as:

$$
P_{+}=\frac{\left(\vec{P}_{1}(1)+\vec{P}_{2}(1)\right) \mathbf{1}}{\left(\vec{P}_{1}(1)+\vec{P}_{2}(1)\right) \mathbf{1}+\vec{K}^{\prime}(1) \mathbf{1}}
$$

Let $\Lambda^{-1}$ be an average interdeparture time, i.e. interval between customers' departure epochs. For the system $B M A P / S M / 1$ without disasters it is known from [27] that

$$
\Lambda=\lambda
$$

where $\lambda$ is a fundamental arrival rate. Because in our system with disasters the departure of batches of unserved customers appear, the equation (26) is not valid here. Using the theory of renewal processes we derive the following result.

Theorem 4. The average interdeparture time $\Lambda^{-1}$ is determined by

$$
\Lambda=\frac{\lambda^{(1)}}{\left(\vec{P}_{1}(1)+\vec{P}_{2}(1)+\vec{K}^{\prime}(1)\right) \mathbf{1}+\left(\lambda^{(1)}-\lambda^{(2)}\right) \vec{P}_{2}(1) \vec{b}_{2}^{(1)} \mathbf{1}},
$$


where the matrix $\bar{b}_{2}^{(1)}$ is defined as

$$
\begin{aligned}
& \bar{b}_{2}^{(1)}= \\
& \int_{0}^{\infty} \mathrm{e}^{D^{(2)}(1) t} \otimes \mathrm{e}^{F_{0}^{(2)} t} \otimes t \mathrm{~d} B^{(2)}(t)+\int_{0}^{\infty} \mathrm{e}^{D^{(2)}(1) t} \otimes\left(t \mathrm{e}^{F_{0}^{(2)}} F_{1}^{(2)}\right) \otimes\left(P^{(2)}-B^{(2)}(t)\right) \mathrm{d} t .
\end{aligned}
$$

\section{Queue length AT AN ARBItrary time}

Introduce into consideration the following stationary state probabilities:

$$
\begin{aligned}
& \alpha(i, \nu, \eta, m)=\lim _{t \rightarrow \infty} P\left\{i_{t}=i, \nu_{t}=\nu, \eta_{t}=\eta, m_{t}=m\right\} \\
& i \geq 0, \quad \nu=\overline{0, W}, \quad \eta=\overline{0, W}, \quad m=\overline{1, M},
\end{aligned}
$$

and vectors

$$
\begin{aligned}
\vec{\alpha}(i, \nu, \eta) & =\{\alpha(i, \nu, \eta, 1), \cdots, \alpha(i, \nu, \eta, M)\}, \\
\vec{\alpha}(i, \nu) & =\{\vec{\alpha}(i, \nu, 0), \cdots, \vec{\alpha}(i, \nu, N)\} \\
\vec{\alpha}_{i} & =\{\vec{\alpha}(i, 0), \cdots, \vec{\alpha}(i, W)\}
\end{aligned}
$$

The vectors $\vec{\alpha}_{i}, i \geq 0$ characterize the presence of $i$ customers at an arbitrary epoch. Using the known expression for average interdeparture time and the same technique as in [27], the relations of $\vec{\alpha}_{i}$ and $\vec{\pi}_{i}, i \geq 0$ can be found. Denote by $\tilde{B}^{(r)}(t)$ the diagonal matrix with diagonal entries defined by the vector $B^{(r)}(t) \mathbf{1}, r=\overline{1,2}$.

Theorem 5. The vector generating function $\vec{\alpha}(z)=\sum_{i=0}^{\infty} \vec{\alpha}_{i} z^{i}$ is expressed in terms of the generating functions $\vec{\Pi}_{1}(z)$ and $\vec{\Pi}_{2}(z)$ as follows:

$$
\begin{aligned}
\vec{\alpha}(z)=\Lambda \vec{\pi}_{0}\left[\left(-D_{0}^{(1)} \oplus F^{(1)}(1)\right)^{-1} \otimes I_{M}\right]+ & \Lambda\left\{\vec{\pi}_{0}(\Psi(z)-I)\right. \\
& \left.+\vec{\Pi}_{1}(z)\right\} B_{1}^{*}(z)+\Lambda \vec{\Pi}_{2}(z) B_{2}^{*}(z),
\end{aligned}
$$

where $B_{r}^{*}(z)=\int_{0}^{\infty} \mathrm{e}^{\left(D^{(r)}(z) \oplus F_{0}^{(r)}\right) t} \otimes\left(I_{M}-\tilde{B}^{(r)}(t)\right) \mathrm{d} t, r=\overline{1,2}$. 
Proof. Using the technique of Neuts [28], we derive the following expressions for the vectors $\vec{\alpha}_{i}, i \geq 0$ :

$$
\begin{aligned}
\vec{\alpha}_{0}=\Lambda & \vec{\pi}_{0} \int_{0}^{\infty} \mathrm{e}^{\left(D_{0}^{(1)} \oplus F^{(1)}(1)\right) t} \otimes I_{M} \mathrm{~d} t=\Lambda \vec{\pi}_{0}\left[\left(-D_{0}^{(1)} \oplus F^{(1)}(1)\right)^{-1} \otimes I_{M}\right], \\
\vec{\alpha}_{i}=\Lambda & \vec{\pi}_{0} \int_{0}^{\infty} \int_{0}^{t} \mathrm{e}^{D_{0}^{(1)} v} \sum_{k=1}^{i} D_{k}^{(1)} \otimes \mathrm{e}^{F^{(1)}(1) v} \otimes I_{M} \mathrm{~d} v \\
& \times P^{(1)}(i-k, t-v) \otimes \mathrm{e}^{F_{0}^{(1)}(t-v)} \otimes\left(I_{M}-\tilde{B}_{1}(t-v)\right) \mathrm{d} t \\
& +\Lambda \sum_{k=1}^{i} \vec{\pi}_{k} \int_{0}^{\infty} P^{(1)}(i-k, t) \otimes \mathrm{e}^{F_{0}^{(1)}} t \otimes\left(I_{M}-\tilde{B}_{1}(t)\right) \mathrm{d} t, i=\overline{1, j} \\
\vec{\alpha}_{j+l}=\Lambda & \vec{\pi}_{0} \int_{0}^{\infty} \int_{0}^{t} \mathrm{e}^{D_{0}^{(1)} v} \sum_{k=1}^{j+l} D_{k}^{(1)} \otimes \mathrm{e}^{F^{(1)}(1) v} \otimes I_{M} \mathrm{~d} v \\
& \times P^{(1)}(j+l-k, t-v) \otimes \mathrm{e}^{F_{0}^{(1)}(t-v)} \otimes\left(I_{M}-\tilde{B}_{1}(t-v)\right) \mathrm{d} t \\
& \left.+\Lambda \sum_{k=1}^{j} \vec{\pi}_{k} \int_{0}^{\infty} P^{(1)}(j+l-k, t) \otimes \mathrm{e}^{F_{0}^{(1)}} t \otimes\left(I_{M}-\tilde{B}_{1}(t)\right)\right) \mathrm{d} t \\
& \left.+\Lambda \sum_{k=1}^{l} \vec{\pi}_{j+k} \int_{0}^{\infty} P^{(2)}(l-k, t) \otimes \mathrm{e}^{F_{0}^{(2)}} t \otimes\left(I_{M}-\tilde{B}_{2}(t)\right)\right) \mathrm{d} t, l>0 .
\end{aligned}
$$

Multiplying (28) by the corresponding degrees of $z$, summing up and using the notion (7), we derive the required expression (27). Theorem 5 is proved.

\section{Calculating the value of the cost CRiterion}

Having calculated the stationary state distribution under the fixed value of the threshold $j$ we can calculate the value of the cost criterion (1).

The formula for the calculation of the mean queue length is determined by

$$
L=\left(\vec{\Pi}_{1}^{\prime}(1)+\vec{\Pi}_{2}^{\prime}(1)\right) \mathbf{1}
$$

Since $\vec{\pi}_{i}=\vec{p}_{i}, i \geq 1$ the following equations are valid: $\vec{\Pi}_{i}^{\prime}(1)=\vec{P}_{i}^{\prime}(1), i=\overline{1,2}$. So we can use the equations (25) for calculating the value of $L$.

By exploiting the ergodic theorems for Markov chains, see e.g. Skorohod [32], it can be shown that

$$
\Phi_{2}=\Lambda \vec{P}_{2}(1) \bar{b}_{2}^{(1)} \mathbf{1}, \quad \Phi_{1}=1-\Phi_{2}
$$

The value of $R$ is calculated by the formula

$$
R=\left(\lambda^{(1)} \Phi_{1}+\lambda^{(2)} \Phi_{2}\right)\left(1-P_{+}\right) .
$$


Substituting (29)-(31) into (1) we get the value of the cost criterion (1). Having of possibility to calculate the value of criterion (1) for any fixed $j$ practically we can find the optimal value of threshold $j^{*}$, minimizing the value of the cost criterion.

\section{NUMERICAL EXAMPLE}

In this section, to illustrate obtained results we present simple numerical examples.

Let the customers input flow be ordinary and we set

$$
\begin{array}{ll}
D_{0}^{(1)}=\left(\begin{array}{cc}
-4.4 & 2.4 \\
2.8 & -7.8
\end{array}\right), & D_{1}^{(1)}=\left(\begin{array}{ll}
2 & 0 \\
0 & 5
\end{array}\right), \\
D_{0}^{(2)}=\left(\begin{array}{cc}
-2.2 & 1.2 \\
4.8 & -7.8
\end{array}\right), & D_{1}^{(2)}=\left(\begin{array}{ll}
1 & 0 \\
0 & 3
\end{array}\right) .
\end{array}
$$

The intensities of the input flow are equal 3.38 and 1.39 for the first and second modes, respectively.

We also assume that the kernel $B^{(r)}(t)$, that describes the service process in the $r$ th mode, has the form

$$
B^{(r)}(t)=\operatorname{diag}\left\{B_{1}^{(r)}(t), \ldots, B_{M}^{(r)}(t)\right\} P^{(r)}, r=\overline{1,2} .
$$

Here $\operatorname{diag}\left\{c_{1}, \ldots, c_{M}\right\}$ denotes a diagonal matrix with the diagonal entries $c_{1}, \ldots$, $c_{M} ; P^{(r)}$ is a stochastic matrix and $B_{i}^{(r)}(t), i=\overline{1, M}$ are distribution functions.

The form (32) of semi-Markovian kernel $B^{(r)}(t)$ means that the sojourn times of service directing process $m_{t}$ depend only on the current state of the process and do not depend on future states. The matrix $P^{(r)}=B^{(r)}(+\infty)$ determines the transition probabilities of the embedded Markov chain $m_{n}, n \geq 1, r=\overline{1,2}$.

Let $M=2$ and $T_{m}^{(r)}$ be the customer service time when the system operates in the $r$ th mode and service directing process $m_{t}$ is on the state $m, m=\overline{1, M}, r=\overline{1,2}$. We assume that

$$
T_{1}^{(1)}=0.15, T_{2}^{(1)}=0.2, T_{1}^{(2)}=0.4, T_{2}^{(2)}=0.3, \quad P^{(1)}=P^{(2)}=\left(\begin{array}{cc}
0.6 & 0.4 \\
0.35 & 0.65
\end{array}\right) .
$$

The average service times are equal 0.176 and 0.346 for the first and second modes, respectively.

For disasters flow we set $N=1$ and

$$
\begin{array}{ll}
F_{0}^{(1)}=\left(\begin{array}{cc}
-0.25 & 0.15 \\
0.24 & -0.33
\end{array}\right), & F_{1}^{(1)}=\left(\begin{array}{cc}
0.1 & 0 \\
0 & 0.09
\end{array}\right), \\
F_{0}^{(2)}=\left(\begin{array}{cc}
-0.21 & 0.16 \\
0.27 & -0.35
\end{array}\right), & F_{1}^{(2)}=\left(\begin{array}{cc}
0.05 & 0 \\
0 & 0.08
\end{array}\right) .
\end{array}
$$




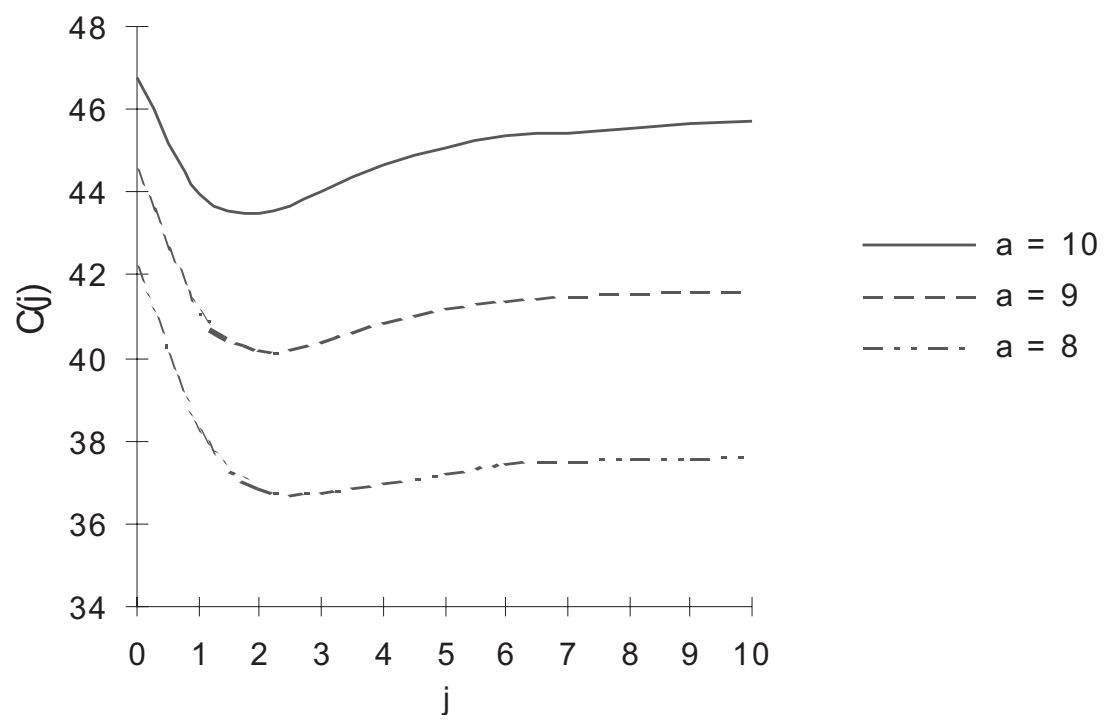

Figure 1. The dependence of the cost criterion value on the threshold.

TABLE 1.

\begin{tabular}{|c|c|c|c|c|}
\hline$a$ & $j^{*}$ & $C\left(j^{*}\right)$ & $C_{1}$ & $C_{2}$ \\
\hline 8 & 3 & 36.75 & 37.73 & 54.01 \\
\hline 9 & 2 & 40.18 & 41.74 & 55.22 \\
\hline 10 & 2 & 43.51 & 45.8 & 56.33 \\
\hline
\end{tabular}

The disasters arrival intensities are equal 0.096 and 0.061 for the first and second modes, respectively. The cost coefficients are defined as: $c_{1}=5, c_{2}=40, g=2.5$.

Figure 1 illustrates the dependence of the cost criterion value (1) on threshold $j$ under some values of the cost coefficient $a$.

Let $j^{*}$ be the optimal threshold value and $C_{r}$ be the cost criterion value when the system operates only in the $r$ th mode, $r=\overline{1,2}$. The values $j^{*}, C\left(j^{*}\right), C_{1}, C_{2}$ under some values of the coefficient $a$ are adduced at the Table 1 .

So the system control allows to reduce the expense of the system operation.

For the following example we consider a batch input flow of customers, that is defined by the following matrices:

$$
\begin{aligned}
& D_{0}^{(1)}=\left(\begin{array}{cc}
-1.45 & 0.45 \\
0.6 & -2.6
\end{array}\right), \quad D_{1}^{(1)}=D_{2}^{(1)}=\left(\begin{array}{cc}
0.5 & 0 \\
0 & 1
\end{array}\right), \\
& D_{0}^{(2)}=\left(\begin{array}{cc}
-2 & 1 \\
0.9 & -1.4
\end{array}\right), \quad D_{1}^{(2)}=D_{2}^{(2)}=\left(\begin{array}{cc}
0.5 & 0 \\
0 & 0.25
\end{array}\right) .
\end{aligned}
$$

The intensities of the input flow are equal 2.14 and 1.1 for the first and second modes, respectively. 


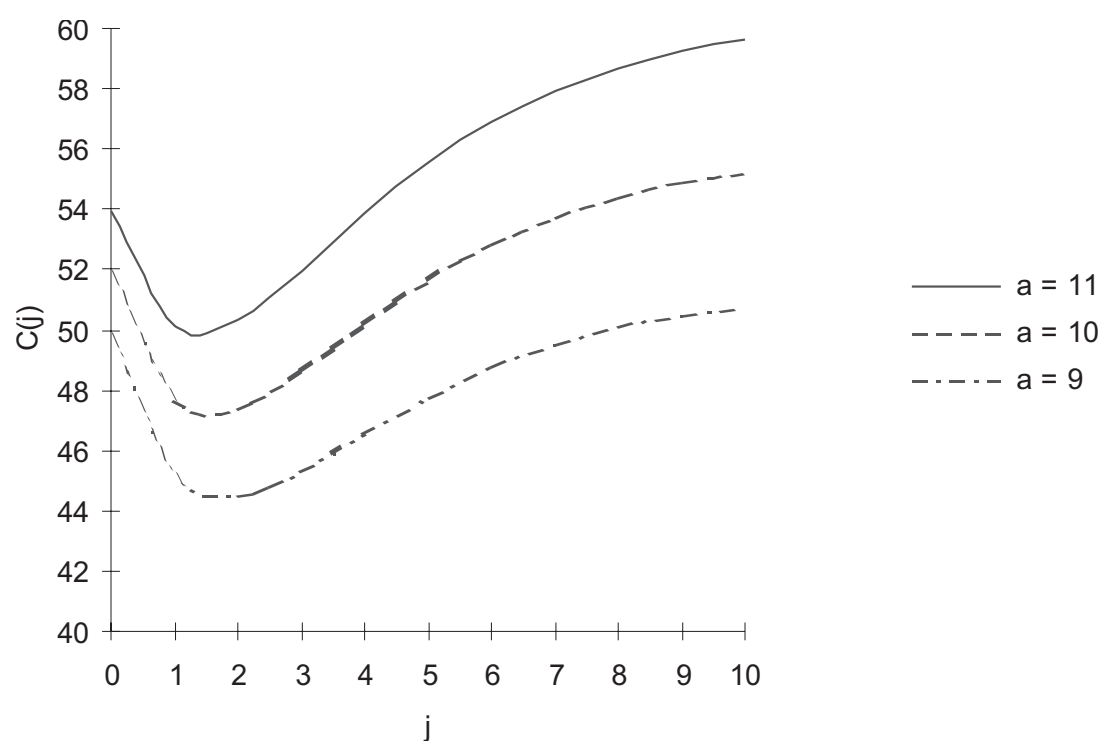

Figure 2. The dependence of the cost criterion value on the threshold.

\section{TABLE 2.}

\begin{tabular}{|c|c|c|c|c|}
\hline$a$ & $j^{*}$ & $C\left(j^{*}\right)$ & $C_{1}$ & $C_{2}$ \\
\hline 9 & 2 & 44.49 & 51.42 & 93.57 \\
\hline 10 & 2 & 47.46 & 54.93 & 94.52 \\
\hline 11 & 1 & 50.12 & 60.44 & 95.45 \\
\hline
\end{tabular}

Let $M=1, \quad T_{1}^{(1)}=0.3, T_{1}^{(2)}=0.25$. The input of disasters is the same as in the previous example. The cost coefficients are the following: $c_{1}=10, c_{2}=85$, $g=5$.

Figure 2 gives the dependence of the cost criterion value on threshold $j$ and Table 2 contains the values of $j^{*}, C\left(j^{*}\right), C_{1}, C_{2}$ under some values of the parameter $a$.

\section{Conclusion}

Controlled queueing system with additional disaster input and two operation modes has been studied. Stationary distribution of system state probabilities has been found and the problem of optimization of the threshold strategy has been solved.

Acknowledgement. The author would like to express sincere thanks to her supervisor A.N. Dudin for a suggesting this problem, a number of helpful comments and for help to improve this paper. 


\section{REFERENCES}

[1] J. Artalejo, G-networks: A versatile approach for work removal in queueing networks. Eur. J. Oper. Res. 126 (2000) 233-249.

[2] A. Chen and E. Renshaw, The $M / M / 1$ queue with mass exodus and mass arrivals when empty. J. Appl. Prob. 34 (1997) 192-207.

[3] A.N. Dudin, Optimal control for a $M^{x} / G / 1$ queue with two operation modes. Prob. Eng. Inform. Sci. 11 (1997) 225-265.

[4] A.N. Dudin and S. Nishimura, Optimal control for a $B M A P / G / 1$ queue with two service modes. Math. Prob. Eng. 5 (1999) 255-273.

[5] A.N. Dudin and A.V. Karolik, BMAP/SM/1 queue with Markovian input of disasters and non-instantaneous recovery. Perform. Eval. 45 (2001) 19-32.

[6] A.N. Dudin and S. Nishimura, A $B M A P / S M / 1$ queueing system with Markovian arrival of disasters. J. Appl. Prob. 36 (1999) 868-881.

[7] A.N. Dudin and S. Nishimura, Embedded stationary distribution for the $B M A P / S M / 1 / N$ queue with disasters, Queues: Flows Syst. Networks 14 (1998) 92-97.

[8] H.R. Gail, S.L. Hantler, M. Sidi and B.A. Taylor, Linear independence of root equations for $M / G / 1$ type of Markov chains. Queue. Syst. 20 (1995) 321-339.

[9] H.R. Gail, S.L. Hantler and B.A. Taylor, Spectral analysis of $M / G / 1$ and $G / M / 1$ type Markov chains. Adv. Appl. Prob. 28 (1996) 114-165.

[10] E. Gelenbe, Réseaux stochastiques ouverts avec clients négatifs et positifs, et réseaux neuronaux. C. R. Acad. Sci. Paris II 309 (1989) 979-982.

[11] E. Gelenbe, Random neural networks with positive and negative signals and product form solution. Neural Comput. 1 (1989) 502-510.

[12] E. Gelenbe, Réseaux neuronaux aléatoires stables. C. R. Acad. Sci. 310 (1990) 177-180.

[13] E. Gelenbe, Stable random neural networks. Neural Comput. 2 (1990) 239-247.

[14] E. Gelenbe, Queueing networks with negative and positive customers. J. Appl. Prob. 28 (1991) 655-663.

[15] E. Gelenbe, P. Glynn and K. Sigman, Queues with negative arrivals. J. Appl. Prob. 28 (1991) 245-250.

[16] E. Gelenbe and S. Tucci, Performances d'un systeme informatique dupliqué. C. R. Acad. Sci. Paris II 312 (1991) 27-30.

[17] E. Gelenbe and M. Schassberger, Stability of product form G-networks. Proba Eng. Inform. Sci. 6 (1992) 271-276.

[18] E. Gelenbe, G-networks with instantaneous customer movement. J. Appl. Prob. 30 (1993) 742-748.

[19] E. Gelenbe, G-networks with signals and batch removal. Prob. Eng. Inform. Sci. 7 (1993) 335-342.

[20] E. Gelenbe, G-networks: An unifying model for queueing networks and neural networks. Ann. oper. Res. 48, (1994) 141-156.

[21] J.M. Fourneau, E. Gelenbe and R. Suros, G-networks with multiple classes of positive and negative customers. Theoret. Comput. Sci. 155 (1996) 141-156.

[22] E. Gelenbe and A. Labed, G-networks with multiple classes of signal and positive customers. Eur. J. Oper. Res. 108 (1998) 293-305.

[23] A. Graham, Kronecker Products and Matrix Calculus with Applications. Ellis Horwood, Chichester, UK (1981).

[24] P.G. Harrison and E. Pitel, The $M / G / 1$ queue with negative customers. Adv. Appl. Prob. 28 (1996) 540-566.

[25] G. Jain and K. Sigman, A Pollaczeck-Khinchine formula for $M / G / 1$ queues with disasters. J. Appl. Prob. 33 (1996) 1191-1200.

[26] D.M. Lucantoni, New results on the single server queue with a batch Markovian arrival processes. Stoch. Mod. 7 (1991) 1-46. 
[27] D.M. Lucantoni and M.F. Neuts, Some steady-state distributions for the $B M A P / S M / 1$ queue. Stoch. Mod. 10 (1994) 575-598.

[28] M.F. Neuts, Structured Stochastic Matrices of $M / G / 1$ Type Applications. Marcel Dekker, New York (1989).

[29] S. Nishimura and J. Jiang, An $M / G / 1$ vacation model with two service modes. Prob. Eng. Inform. Sci. 9 (1995) 355-374.

[30] R.D. Nobel, A regenerative approach for an $M^{X} / G / 1$ queue with two service modes. Automat. Control Comput. Sci. 32 (1998) 3-14.

[31] R.D. Nobel and H. Tijms, Optimal control for a $M^{X} / G / 1$ queue with two service modes. Eur. J. Oper. Res. 113 (1999) 610-619.

[32] X. Skorokhod, Probability Theory and Random Process. High School, Kiev (1980).

[33] H. Tijms, On the optimality of a switch-over with exponential controlling the queue size in a $M / G / 1$ queue with variable service rate. Lect. Notes Comput. Sci. (1976).

To access this journal online: www.edpsciences.org 